P.

THE EFFECTS OF EVACUATING THE

\title{
AQUEOUS HUMOR
}

IN

INFLAMMATION OF THE EYES;

AND IN

SOME DISEASES OF THE CORNEA.

BY JAMES WARDROP, F.R.S. ED.

Read May 11 and 25, 1813.

IN January, 1807, some cases of Ophthalmia were published in the Edinburgh Medical and Surgical Journal, in which the evacuation of the aqueous humor was attended with beneficial effects. At that time, the practice was to be considered only in its infancy, the number of cases in which it had been tried were very limited, and I was unable to point out, with any degree of precision, the particular species of Ophthalmia, and those symptoms of the disease which could be relieved by this mode of treatment. I had, however, sufficient experience to be convinced, that 
the operation could be performed, in almost every case, without aggravating, if it cid not arrest the progress of the inflammatery symptoms. I therefore embraced that channel of laying my observations before the public, that both the application and the utility of the practice might be established by the additional experience of others.

The subsequent successful result of this operation in a series of cases of Ophthalmia, and its utility in some affections of the Cornea, have induced me to bring the subject before the public in this Memoir; being now enabled to point out, with some confidence, the particular species of the disease, and those symptoms, which the evacuation of the Aqueous Humor is best calculated to remove, and, to recommend it as a practice, from which important advantages may be derived in some violent and alarming cases.

\$1. General Observations on the Evacuation of the. Aqueous Humor.

I formerly took notice*, that I was first led to evacuate the Aqueous 'Humor in some of' the diseases, of the eye from a very curious phænomenon observed by Dr. Barclay in that organ after death. He remarked, that if the eye of an animal be

* Edin. Med. and Surg. Journal, vol. III. p. 56. 
moderately squeezed in the hand, the whole cornea will instantly become cloudy, and when the pressure is increased, the obscurity is also increased. If it be still more squeezed, the cornea becomes of such an opake, milky colour, that the iris cannot be distinguished through it. He likewise observed the same appearances produced from filling the veins with water or quicksilver; and, whenever the pressure or over-distending cause was removed, the cornea completely regained its transparency, and appeared as if no such experiment had been made*.

- From this curious phænomenon in the dead eye, it was probable that, in the living, the transparency of the cornea might vary according to the degree of its distension; and that, in some varie ties of opacities of the cornea, the obscurity might arise from an increase in the quantity of the con. tents of. the eyeball. Besides, therefore, bloodletting, purging, and the other means usually recommended in the treatment of the inflammation which generally accompanies obscurities of the cornea during the early periods of the disease, it occurred to me, that a more complete and sudden diminution in the contents of the eyeball might be produced by evacuating the aqueous humor. :

* See the 'Muscular Motions of the Human Body, by Jolm Barclay, M. D. 
A favourable case for the trial of this operation soon occurred, where there was a very considerable degree of milkiness and opacity of the cornea, and in which also the eyeball appeared also distended, prominent, and accompanied with acute inflammatory symptoms. I discharged the aqueous humor by a small incision through the cornea, and had the satisfaction to find that the operation produced not only an alteration in the degree of transparency of the cornea, but also that the pain, and all the inflammatory symptoms, were removed.

From the success of this case, I was not only convinced of the good effects which this operation might have in removing some opacities in the cornea, but, from the unlooked-for alleviation of the inflammatory symptoms, I was afterwards led to have recourse to this mode of treatment in violent cases of inflammation of the eyeball, in which the cornea had little or no share in the disease.

The evacuation of the Aqueous Humor in Ophthalmia may appear to some, on first considering the subject, a violent and even formidable mode of practice, from the means necessary to accomplish it, consisting in a wound made in an organ already highly inflamed and become extremely irritable and painful. But the pain of making a puncture through the cornea is by no means 
acute, when the cornea itself is not inflamed, or otherwise diseased; and in the operation for the extraction of the cataract, where the cornea is quite sound, I have heard patients compare the sensation of making the incision through to a hair drawn across the eye. Though the cornea, in the natural state, has but little sensibility, yet it should be observed, that, when diseased, it becomes the seat of very considerable pain; and when it is inflamed, or ulceration has taken place, the pain of an incision then becomes acute. Besides, from the irritation which the exposure to light produces in an inflamed eye, and more or less pressure being always necessary to hold the eyeball steady, the evacuation of the aqueous humor cannot be accomplished, in many cases, without giving a considerable degree of uneasiness. The pain, however, produced by'the operation, particularly if it be done with care and attention, soon subsides, and the good effects which quickly succeed, sufficiently compensate for any uneasiness it may have occasioned. Wounds, too, of the carnea heal with uncommon rapidity; and I have not, in a single instance where the operation has been performed, been able to detect the vestige of an incision; nor has it ever oc. curred, so far as I know, that any visible cicatrix remained, even in those cases in which the operation had been performed when the cornea was in a previous state of ulceration. 
In those cases where the practice of evacuating the aqueous humor is judiciously employed, although the operation may create some temporary irritation, yet its good effects will become immediately perceptible, and in most cases will be permanent. The more obvious of these are a more or less considerable improvement in vision, particularly in those cases where there is a cloudiness in the anterior chamber;-a complete cessation of the sense of fulness of the eyeball, and pain in the head;-and, in some cases, a very remarkable change in the size of the inflamed vessels,

The great and immediate relief which is obtained by the evacuation of the Aqueous Humor in ophthalmia, most probably arises chiefly from the sudden removal of tension. The pain accompanying inflammation in other organs of the body is, in general, in proportion to the degree of tension and resistance of the adjacent parts. It is well known how much relief is afforded in deep-seated inflammations, such as that of the periosteum, and in some varieties of whitloe, by making a free incision through the skin and external parts. The same thing is also illustrated by the good effects produced from dividing the gums during dentition, and the relief generally afforded by the evacuation of the matter of an abscess. In the eye itseif this observation is very strikingly applicable; for it often happens, in violent cases of ophthalmia, 
that suppuration takes place within the globe' and the purulent matter and humors are suddenly discharged by the coats of the eye bursting. Whenever this takes place, it is invariably remarked, that all the inflammatory symptoms are suddenly alleviated.

There is a case mentioned by Prochaska *, where the aqueous humor was so acrid as to tar. nish the cataract knife; yet this change does not appear ever to take place in ophthalmia.

In those cases where the discharge of the aqueous humor has been found beneficial, it is not even necessary to suppose that its natural quantity is increased. The inflammation will add both to the number and size of the blood-vessels within the eyeball, and consequently will increase the quantity of its contents. If therefore the pain and any of the other symptoms arise, or are aggravated by that unnatural distension, the same good effects will result, whether the contents of the eyeball be lessened by a diminution in the number and size of the blood-vessels, or by the discharge of the aqueous humor. In many cases of ophthalmia, it is very probable that either of these means would have the effect of abating the

- Vide Voigtel Handbuch von der Pathologischen Anatomie, II Band. p. 110. 
violence of the inflammatory symptoms; that in some cases the one shall be useful, whilst the other fails; and that, in very violent cases, both means may be employed at the same time with much advantage. It is not, therefore, to be understood, that the discharge of the Aqueous Humor is here to be recommended as the sole remedy in any case of ophthalmia, but is only to be considered as a powerful auxiliary in some, and in others as a sure, and perhaps the only means of preventing a total destruction of the organ.

When the object is to diminish suddenly the contents of the eyeball, the evacuation of the aqueous humor must fulfil this intention in a more complete manner than can be conceived probable by any means of abstracting blood from its vessels; for as the ophthalmic artery comes from the encephalon, little blood can be taken directly from any of its branches, and it would require a great quantity of blood to be drawn from the temples or neighbouring arteries, to make any remarkáble change in the quantity of the contents of the eyeball; or, at least, a change equal to that which would be produced by the discharge of the aqueous humor. From the advan. tages also which have been universally found to arise from a sudden depletion of blood, in comparison with what can be derived from a slow detraction, considerable benefit might be expected from 
the practice now proposed; for as its effects must be immediate, a sudden change will be produced in the state of the organ, and a change favourable to the abatement of the inflammatory symptoms.

\section{\$2. Of those Cases of Ophthalmia rohich are relieved} by the Evacuation of the Aqueous Humor.

From the number and variety in the phænomena which accompany Inflammation of the Eyes, the combinations and various modifications of those phænomena, and the fruitless attempts which have been made to describe the different species of the disease, it becomes difficult to point out with precision, in this place, those particular forms of it, in which the propriety of discharging the aqueous humor is indicated. It would be foreign to the object of the present Memoir to enter into any elaborate description of the different species of inflammation of the eyes, my intention being to attempt this in a future publication. It will now be sufficient to remark, in general, that those parts composing the organ of vision, in which there is a difference in the natural structure, present different phænomena when they become inflamed. Inflammation of the conjunctiva or mucous mem. brane of the eye is accompanied with that puriform discharge so characteristic of all inflamed mucous surfaces, as that of the urethra, vagina, bronchiæ, nose, and every other surface of a similar nature in the body. Inflammation of the differ. 
ent struetures which compose the corned, of the iris, of the choroid coat, of the sclerotic coat, and of its investing cellular membrane, all present a variety of distinct and characteristic symptoms. Besides this difference, arising from a difference in the natural structure of the parts affected, there are distinct species of inflammation which derive their particular characters from being the effect of specific diseases. Of these, scrophula; cancer, lues venerea, rheumatism; and gout; afford examples; all which occasionally attack the eyes, in common with most other organs of the body. In all these species of ophthalmia, particular cases do occur, in which the aqueous humor may be discharged with ad, vantage; for though the inflammation almost al. ways originates, and is most severe, in one tex. ture ; yet the whole eye is in many cases more or less affected, and thus symptoms arise which the evacuation of the aqueous humor is well calculated to remove.

I shall therefore, after describing the mode of performing the operation, enumerate those forms of Ophthalmia in which the practice has been found beneficial, and illustrate the observations with the history of some cases. Before, however, entering into, this detail, I may observe, in general, that there are no states of the eye in which this treat, ment is :so applicable as in those cases where the coats threaten to give way ; for, as was before 
mentioned, many authors who have described ophthalmia, have particularly noticed, that when the eye bursts, whether from internal suppuration, or from ulceration of the cornea, a remarkable alleviation takes place in the pain, and an abatement in all the other inflammatory symptoms. In the puriform, or, as it is often called, the Fgyptian ophthalmia, this is the usual termination of the severe cases of the disease, and is that change which, by producing a collapse of the globe of the eye, renders the organ irrecoverable.

But besides these, there are cases of a very different description; cases in which the evacuation of the aqueous humor is of much advantage, and where the disease, though not of such a dangerous nature, is yet found unmanageable by the means usually employed. The particular cases alluded to, are those in which the cornea and anterior chamber acquire a peculiar kind of dimness, the transparency being restored; and the accompanying inflammatory symptoms alleviated, by the dis. charge of the aqueous humor.

Besides the peculiar muddiness in the anterior chamber, these cases are characterised by an uneasy feeling of distension in some part of the head, chiefly the forehead; and this symptom generally yrelds to the evacuation of the aqueous humor, 
5 3. Of the Mode of discharging the Aqueous Humor,

The aqueous humor may be discharged by a very simple operation, nothing more being ne. cessary than to make an opening through the cornea of a sufficient size to allow the fluid to escape, and in such a situation that any subse. quent cicatrix may not impair vision,

The opening may be made with any of the knives commonly used for extracting the cataract. It is sufficient that the point of the knife be introduced through the cornea so that it makes a puncture into the anterior chamber; and this should be done near its junction with the sclerotic coat, at any part of the circumference. When the knife has penetrated the anterior chamber, it may then be withdrawn a little, and the blade turned on its axis, so that the aqueous humor will readily escape; and it is better not to remove the instrument altogether, till the fluid is observed to be discharged; for if the incision be not sufficiently large, and the knife taken away before the aqueous humor flows out, the elasticity of the cornea closes the wound, and either hinders the evacuation from being so sudden, and consequently so efficacious, or the closure of the wound entirely prevents its escape. The operation, therefore, which is necessary to discharge the aqueous humor, is merely the first step of the section of the cornea, made in extract- 
ing the cataract, or what has been called the punctuation of the cornea.

The chief difficulty in performing the operation arises from the pain occasioned by pressure on the eyeball, necessary to keep open the eyelids ; for until a sufficient portion of cornea is brought into view, and the movements of the eyeball completely under the management of the operator, the introduction of the knife should not be attempted.

The upper eyelid should be kept open either by the fingers of an assist ant, or by Pellier's speculum. If this instrument be employed, it will be found useful to have the silver wire covered with a piece of crape, which will prevent it slipping from any moisture of the skin, an accident very common, and very troublesome. The operator, with the fore and middle fingers of one hand presses down the under eyelid, and applies their points over the tarsi, in such a manner that they touch the eyeball, and can apply any degree of pressure upon it which may be necessary. After the assistant raises the upper eyelid, the patient should be directed to look downwards; and then the assistant employs sufficient pressure, to keep the eye in that position.

The operator may then make the puncture; but as the patient is very apt to start when he first finds 
the instrument coming in contact with his eye, I have found it useful, merely to touch the cornea repeatedly with the back of the knife till all risk of starting is over; and as soon as the back of the point of the instrument rests on the part where the puncture is to be made, the knife can be raised very steadily on its point; and then thrust into the anterior chamber.

Though I have described the method by which the puncture of the cornea may be made with a common extracting knife, yet it is evident that the aqueous humor may be discharged equally well by other instrumentsieand I have often employed the needle of $\mathrm{Mr}$. Cheselden. The more we are in the habit of using any particular instrument, the more dexterity and ease are acquired in its use.

54. Of the Evacuation of the Aqueous Humor in the Puriform Ophthalmia.

There is no fact more striking in the history of the Puriform Ophthalmia, than the immediate and permanent relief which many of the unfortunate sufferers experienced, when the eyeball burst, and part of its contents was discharged. During the most excruciating pain of the eye and head, their agony was suddenly relieved when this accident took place, which in some cases was even accompanied by a cracking noise. This is particularly 
taken: notice of by, those who have related the history of the disease, and it will be found a striking incident in all the accounts given by patients themselves, in whom the organization of the eye is found to be destroyed *.

An artificial mode of diminishing the contents of the eyeball might, in such cases, be naturally expected, not only to produce the same alleviation of the violent symptoms of the disease, but by preventing the rupture of the cornea, and the subsequent protrusion of the iris, which takes place, would save the organ from that total destruction which is almost the inevitable consequence of the natural progress of the disease. The evacuation of the aqueous humor is well calculated to fulfil this purpose, and in a very considerable number of cases has been followed with the happiest effects. Mr. Ware, whose authority is so well entitled to our respect, first mentioned to me the probable good effects which the evacuation of the aqueous humor might be expected to produce, in those cases where the eyeball shews a tendency to burst, and in some remarks which he afterwards published on the Puriform Ophthalmia this practice is recommended, and two cases given where it was successfully employed. The practice has also been adopted in this species of ophthalmia to a very considerable extent, by Mr. Mac-

* Farrel and Veitch on Ophthalmia, \&c. 
gregor, surgeon to the York asylum". "I have, within the last two years, (Mr. M. remarks) performed this operation in twenty-three instances, with a degree of success that strongly induces me to recommend it. For of this number, twentyone were immediately relieved by it, and afterwards recovered their sight under the usual mode of treatment." And again Mr. M. observes, " It is to be regretted that this operation is not more frequently performed; for I am convinced that many persons have lost their sight froin a rupture of the cornea taking: place at that part opposite the pupil, which a timely.and judicious performance of this operation might have prevented." As in the greater proportion of the cases which have come under my observation, the disease has been particularly mild, I have had no opportunity of reaping the benefits of this operation; for, among several hundred examples, there nas not been one case where the cornea burst, or where suppuration took place within the eyeball: all the more severe symptoms being speedily removed by bleeding, purging, and a strict pursuance of the antiphlogistic regimen.

The more violent cases of this ophthalmia have appeared chiefly in the army, and it is therefore in our military hospitals, where we can expect to find most advantage from this practice.

* Transactions of a Society for the Improvement of Medical and Chirurgical Knòwledge, vol. iii. p. 60. 
In addition, therefore, to the other means commonly employed, more particularly bleeding and purging, the evacuation of the aqueous humor must be considered as a useful remedy in the Puriform Ophthalmia. Its introduction into those countries where the disease is particularly prevalent, and where it appears in the most severe and dangerous forms, would probably be attended with the most beneficial effects, and its judicious employment might be the means of saving the eyes of many of our soldiers who are abroad, more particularly those on the Mediterranean stations.

Little difficulty can occur in selecting the fit cases of the Puriform Ophthalmia, and in determining the proper period for performing the operation. No states of the eye, during the progress of this disease, can arise as far as I know, which could render it improper, provided the accompanying symptoms are in any degree severe, and therefore the practice should be had recourse to, in all cases where the pain in the head and sense of distension in the eyeball are considerable. In those cases where the cornea threatens to burst, the operation becomes particularly urgent, and should be had recourse to without delay. The bursting of the cornea, in some cases, is preceded by an apparent change in the structure of that part of the eye; in other cases no previous alteration can be detected. The changes generally observed are, that the cornea becomes muddy in one part, or over the VOL. IV. 
whole surface; or its surface is studded with small white spots, which run into one another, as the inflammation advances to suppuration; and then ulceration of the cornea to a greater or less extent, always takes place. In other cases the cornea suddenly gives way, without any apparent previous change*. This has been particularly taken notice of by Dr. Veitch, and he remarks that in some cases of this ophthalmia, where he had an opportunity of examining the eye, both immediately before and after the aqueous humor was discharged, this fluid had escaped " by a division of the cornea, which was nearly as clean as if it had been cut with a knife." In the puriform ophthalmia of children, I have had repeated opportunities of making the same remark, having observed a straight division toward the centre of the cornea, the adjacent portion being apparently sound.

\$5. Of the Evacuation of the Aqueous Humor in the Puriform Ophthalmia of Children.

The history, symptoms, and consequences of the puriform ophthalmia in children, are so extremely similar to those of the puriform ophthalmia of the adult, that what has been said of the effects of evacuating the aqueous humor.in the one, may be repeated regarding the other. When the inflam-

* Observations on Ophthalmia, by Charles Farrel, M.D. 
mation arising in the conjunctiva advances to the other textures of the eye, and is not arrested in its progress, the eyeball often bursts by a rupture of the cornea. Now when there is any danger of apprehending such an accident, when an ulcer of the cornea is making rapid progress or when the violence of the inflammatory symptoms indicate the danger of a rupture of the cornea, the aqueous humor may be evacuated, with the hope, not only of alleviating the severe symptoms of the disease, but of preventing that destruction of the cornea which is always followed by a change in the form of the eyeball, -an obscurity of more or less of the cornea, - a displacement of the iris, - and a deformity or destruction of the pupil.

\section{Of the Evacuation of the Aqueous Humor in the Gonorrheal Ophthalmia.}

Though we have no means of arresting the progress of some specific diseases, yet a great deal may be done to mitigate their symptoms. When the eye becomes affected with Gonorrheal Ophthal. mia, the progress of the disease is generally so rapid, and the symptoms so severe, that the most powerful remedies are necessary to prevent its speedy destruction. Besides, therefore, the extensive depletive system, which should in such cases be adopted, it will be of great importance 
to watch the progress of the disease in the anterior chamber, and when any symptoms occur which indicate the danger of a rupture of the cornea, the aqueous humor should be evacuated without delay.

\section{\$ 7. Of the Discharge of the Aqueous Humor, where the Capsule of that Humor is inflamed.}

On a former occasion* I attempted to shew, that each of the three distinct textures which compose the cornea may be separately inflamed. There is no inflammation of the eye, where so much benefit is derived from evacuating the aqueous humor, as when that inflammation affects the internal layer of the cornea or membrane which contains the aqueous humor.

This disease is marked by a muddiness or turbidity of the anterior chamber which an ordinary observer would not readily ascribe either to an opacity of the cornea, or to a diminution in the transparency of the aqueous humor. Besides this diffused cloudiness there are generally one or more defined spots, which distinctly denote an opacity of the cornea. These do not resemble a common speck, but have a mottled appearance, and around the opake, white, central points there is a kind of disk, very like what is perceived in some agates, and

* Essays on the Morbid Anatomy of the Eye, Vol. I. and II. 
which are commonly called the eyes of pebbles. There is not much external redness accompanying the obscure anterior chamber - a blush of vessels only being seen around the sclerotic coat, at that place where the iris is attached to it. Neither does the patient seem to suffer much pain from the effects of light, as he can generally open the eyelids and keep them so, finding no benefit from a shade. The symptoms of this disease which are most distressing, are a sense of fullness and distension of the eyeball, as if it was filled with matter, and a dull agonizing pain, generally either in the brow or back part of the head; or in both these parts.

In this disease I have never found the evacuation of the aqueous humor fail in procuring an immediate relief of the pain of the head, and an instantaneous restoration of the transparency of the anterior chamber.

\section{CASEI.}

A woman, fifty years of age, had a general milkiness of the anterior chamber of the left eye, and a slightly opake spot nearly in the centre of the cornea. On the white of the eye there were a considerable number of distinct red vessels, none of which passed over the cornea. She could distinguish light with this eye, or a dark object placed 
between her and the light; but neither form nor colours. Exposure to light gave her little uneasiness. - Had occasional epiphora, but particularly complained of a dull pain, extending through the head and eyeball.

The affection had lasted about a fortnight. After having had headach during the night, she found the eye red and painful when she awoke, and objects appeared as if seen through a mist. The symptoms had afterwards gradually increased.

Treatment.-I discharged the aqueous humor by a puncture through the cornea; and immediately after the operation she could perceive a finger with a ring on it, which was held before her eye.-She complained of a sensation as if a mote were in the eye, but she felt no acute pain. The cornea immediately regained its natural transparency, except at the speck, which became more distinctly circumscribed.

Some hours after the operation, the pain recurred with considerable violence, but it was relieved by the operation of a purge, fomenting the parts adjacent to the eyeball, and cupping the temples. In a few days the eye was quite recovered. 


\section{$C A S E I I$.}

In a youth fifteen years of age, the anterior chamber of the right eye had lost a good deal of its natural transparency, which appeared to proceed partly from an obscurity of the cornea, and partly from a turbidity of the aqueous humor. There was a bright crimson redness over the whole albuginea; the vessels appeared deep, yet their ramifications were distinct. He complained of a sense of fullness in the eyeball, and of a dull, heavy. pain in the orbit, eyebrow, and extending through the head. The pain of the eye was much increased by exposure to light. The inflammation had existed for three weeks, and had from its commencement been attended with pain in the head.

Treatment.-The pain in the brow and head subsided immediately after the aqueous humor was discharged, and in the course of two days, the redness and obscurity of the anterior chamber went off.

\section{CASE III.}

There was a want of transparency in the anterior chamber of the right eye, which seemed, partly, to proceed from an opacity of the cornea, 
and partly from a turbidness of the anterior chamber.

A bright scarlet-coloured inflammation appeared over the whole albuginea, and the vessels seemed deep, but the ramifications were quite distinct. Exposure to light was very painful, and the patient complained of a sense of fullness in the eyeball, and of a heavy pain in the orbit, and brow, extending through the head. The inflammation had continued three weeks, and from its commencement had been attended with pain in the head.

Treatment.-The aqueous humor was discharged, and immediately afterwards, the pain in the brow and head completely subsided. The smarting from the operation continued only for a few minutes. He could open his eyes more freely. Redness increased, but of a darker shade, and the vessels more tortuous. No pain in the eye.

\section{CASEIV.}

A sailor boy, thirteen years of age, felt an itching sensation in his right eye, which, by constant rubbing, became red and painful. About eight weeks after, he was at sea during a storth of thunder and lightning, which increased the inflammation. The left eye also, at that time, 
became affected. I saw him about ten weeks after the first attack. The anterior chamber of the left eye had become dull and cloudy; and there were three or more milk-white spots on the cornea, of the size of a pin's head, perfectly round, and distinctly circumscribed. The pupil was a little contracted; and there was only a very slight redness of the sclerotica, near to its union with the cornea. There was also a general cloudiness of the cornea of the right eye; and, on the inferior part of it, there was a speck of considerable size, with several red vessels ramified through it. There were also some small opake circular specks on different parts of the cornea. The blood-vessels were more numerous on the sclerotica of this eye than of the left. Exposure to light gave acute pain in both eyes, and made them gush with tears. He had violent pain in both eyes, but particularly in the right; and he described the pain as having been extremely severe the day before, through his whole head. He also felt a sense of distension or weight over the eyebrow, and had slight general fever: A by=stander observed, that his eyes appeared too full.

I made an incision into the cornea of the left eye, and the aqueous humor spirted out forcibly: The operation occasioned smart pain for two or three minutes. A little blood afterwards appenyed in the anterior chamber, which was effused in consequence of the division of the red vessels 
which entered the edge of the cornea.' The operation was afterwards performed on the right eye, and it caused more acute pain than in the left, for the incision was made through a cornea which was highly vascular, and had acquired a morbid degree of sensibility. In a few minutes, all the pain, which he conceived to arise from the cut, subsided; and the uneasy fullness and distension in both eyes were greatly diminished. He was ordered to use fomentations, and to take a purgative medicine. I saw him three days afterwards : he had no return of the pain of the eye or head since the operation : he could endure the light; and his vision was so perfect, that he walked two miles, and could distinguish readily, and without uneasiness, all objects around him. The opacities of the cornea of both eyes were greatly diminished; and there was very slight redness of the sclerotica.

\section{CASEV.}

A healthy-looking man, forty-five years of age, had inflammation in both eyes for six weeks, which began after the abatement of a violent pain of his head. There was a general cloudiness of the cornea of the left eye. The iris could be distinguished but indistinctly. There were numerous vessels on the white of the eye; and they formed clusters 
on different parts, giving it a mottled appearance. The eye looked unnaturally dry; and there was no intolerance of light. The disease in the right eye had the same appearance; but the cloudiness was limited to two-thirds of the cornea; and the red vessels were not so numerous as in the left eye. The vision of the left eye was almost entirely destroyed ; and with the right one, every object appeared as if seen through a mist or smoke. Both eyes looked full and prominent. An eye-water, of corrosive sublimate, and the application of an ointment composed of the red oxyd of mercury, diminished, in a few days, the inflammation and opacity of the right eye. The obscurity of the left cornea, however, increased, so that the iris and pupil were very indistinctly seen through it.

Treatment.-I perforated the left cornea with a spear pointed couching-needle, and immediately it appeared clearer to several by-standers. The operation was attended with no pain, neither was it followed by any inflammation. He afterwards used a wash of the nitrate of quicksilver, and the cornea soon regained its transparency. The pupil, however, remained a little irregular, with a slight dimness behind it; and objects appeared with this eye as if through a mist. By the external application of sulphuric æther, the dimness disappeared; and I saw him eight months afterwards, when he told me that he then saw as well as when he was a boy; for he had been short-sighted before his eyes became 
inflamed; and probably the operation had diminished the natural convexity of the cornea.

\section{Of the Evacuation of the Aqueous Humor in} Abscess of the Anterior Chamber.

In all cases where a puriform fluid is forming in the anterior chamber, the evacuation of the aqueous humor will be found highly useful; for, except in a few remarkable instances, this never occurs where the inflammatory symptoms are mild. In most cases the deposition of a puriform fluid is attended with great pain in the eye and head, and a sense of fullness and tension of the eyeball. When these symptoms are nat subdued by the common methods of treatment, ulceration of the cornea takes place, and the matter, along with the aqueous humor, is discharged, whilst more or less of the iris is protruded through the wound.

The artificial dișcharge of the aqueous humor in the early stages of the disease, alleviates all the inflammatory symptoms, whilst the employment of the same means in the more advanced stages of the complaint, invariably prevents that rupture of the coats of the eye, which in general very much impairs, if it does not entirely destroy its organization.

In some cases the matter deposited in the anterior chamber, has a considerable degree of consist- 
ence and tenacity, and will not be discharged by that puncture which is sufficient to evacuate the aqueous humor. When the matter is in small quantity, it is necessary to discharge the aqueous humor alone, for, -if the inflammatory symptoms be alleviated, the matter will afterwards be speedily absorbed; but in those cases where the quantity collected is considerable, and necessary to be evacuated, it is requisite to make an incision in the cornea nearly equal to that for the extraction of the crystalline lens.

\section{CASE VI.}

A man, forty-five years of age, had a violent inflammation of the whole eyebatl, in consequence of a blow which he received on it three weeks before from a piece of coal, when mining. Nearly one half of the anterior chamber was filled with a puriform fluid. The central part of the cornea was opake, and ulceration was commenced on its surface, and he complained of violent pain both in the eye and head. A semicircular incision was made in the cornea, through which the aqueous humor along with the matter were evacuated. The pain ceased instantly. The wound of the cornea soon healed, no inflammatory symptoms returned, and the cornea regained its transparency by the application of stimulants, leaving only'a small speck. It was evident, that in this case the cornea would have soon 
given way where the ulceration had commenced; by which the organ would have been completely destroyed.

\section{CA SE VII.}

A gentleman, about twenty-one years of age, had a very violent inflammation of the left eyeball. The sclerotica was covered with numerous scarletcoloured blood-vessels; but none of them passed over the transparent cornea. The anterior chamber was turbid ; and several small spots, of a matter resembling pus, were seen in it, towards its circumference. The pupil was much contracted, the eyelids swelled, and their external surface covered with varicose veins. There was a constant flow of acrid tears. Vision was almost entirely destroyed; but, notwithstanding, the eye was extremely sensible to light. He had great pain in the eyeball, and constant headach, with a sense of fullness in the orbit. The inflammation had begun five weeks before, without any known cause, and every symptom had, since that time, gradually increased.

Treatment.-I made a puncture with an extracting-knife through the cornea, and the aqueous humor spirted out. From the difficulty in securing the eyeball, the pressure employed gave consider- 
able pain. The incision smarted for tyo or three minutes; but, before I left the room, he said he found great relief; and that the pain in the eyeball and head, and the peculiar feeling of weight and distension, were entirely removed. On examining the anterior chamber, all the turbidness had disappeared, and the cornea seemed perfectly transparent. He was advised to do nothing but foment the eye and the neighbouring parts, and to take a purge. As he lived at some distance from town, I had no opportunity of seeing him afterwards; but I was informed, that an hour after the operation, all the pain, occasioned by the incision and the pressure, was entirely gone, and the eye had become quite easy. In two days all the redness went off; and I was informed that the mark of the incision could not be discovered; that the inflammation had disappeared; and that his vision was nearly as perfect as it had been before the commencement of the disease. Some months afterwards, I heard that he continued perfectly well.

\section{\$9. Of the Effects of discharging the Aqueous Humor in Staphyloma.}

The effects which are produced by discharging the aqueous humor in some cases of Staphyloma, illustrate in a very satisfactory manner, the mode by which this operation seems to alleviate the symptoms of ophthalmia which have been already 
noticed. For in many cases of Staphyloma whilst the tumor is increasing in bulk, the ball of the eye becomes inflamed, and the patient generally complains not only of a sensation of uneasiness and fullness in the eye itself, but of a pain more or less severe in the brow of the affected side; and under these circumstances the escape of the aqueous humor fails not to produce immediate relief, and none of the inflammatory symptoms recur until a regeneration of that fluid takes place.

The aqueous humor is often evacuated in Staphyloma without the interference of art, the portion of cornea which most easily gives way allowing an exit to that fluid. In several cases I have been able to observe this process frequently take place, so that ultimately a fistulous opening was established, which either remained always pervious, or which was occasionally closed by a thin, pellucid membrane which gave way, wherever a superabundant quantity of fluid was collected in the eyeball.

This natural progress of the disease points out a very easy method of affording relief when the pain and inflammatory symptoms are severe. Under such circumstances, the aqueous humor may be evacuated by puncturing the tumor; an operation which cannot fail to alleviate all the symptoms in as complete a manner as would be fulfilled by that process which nature would ultimately accomplished. 


\section{CASE VIII.}

A gentleman who had a Staphyloma of one eye for some years, produced, in consequence of a severe attack of inflammation, but which, till now, had given him little pain or uneasiness. He was suddenly seized with pain in it, which soon spread to the brow and back part of the head; and it became so agonizing, that in a few hours he was nearly distracted with its severity. He became extremely pale, and his pulse was very feeble.

Treatment.-I discharged the aqueous humor, from which he obtained instant relief; and in a few hours he was perfectly recovered. He was three or four times similarly attacked at the intervals of several weeks. The discharge of the aqueous humor was each time followed with instant and complete relief; and in order to prevent the recurrence of the symptoms, after puncturing the most prominent part of the cornea, I cut out a small circular portion of it. This opening was afterwards covered with a thin pellucid membrane, which readily gave way whenever there was a superabundance of the aqueous humor; and since the opening hath been made, he has remained free from pain. 


\section{CASE IX.}

A woman received a sharp blow on her eye, which was considerably enlarged from a Staphyloma. Two days afterwards the diseased eye-ball was inflamed, extremely sensible to the touch, and she complained of a severe pain in the brow, extending through the head, accompanied by great prostration of strength, sickness, and fever. I punctured the staphyloma, and discharged a bloody fluid, after which she received much relief : the pain in the head disappeared, and the eye became easier. Four days afterwards the pain returned, but complete relief was obtained by again evacuating the contents of the staphyloma.

It is very probable, that in this case all the violent symptoms were produced from an effusion of blood within the eye-ball, in consequence of the injury.

$\$$ 10. Of the Effects of evacuating the Aqueous Humor in Prolapsus of the Iris.

Even after the cornea has given way, and the aqueous humor has been evacuated, in severe cases of ophthalmia, the relief which that discharge produces does not remain permanent; for the portion of iris which is prolapsed, so completely fills o wuride that no more aqueous humor can 
escape, should its quantity become superabundant. Under these circumstances, an artificial discharge not only alleviates the inflammatory symptoms, but, if the protrusion of the iris has been recent, the depletion of the anterior chamber will permit the iris to fall back into its natural situation. In a case of this kind, where Mr. Ware performed the operation, immediately after the evacuation of the aqueous humor, the iris resumed its natural position. Should the iris still continue to be pressed forward, even after a puncture has been made in the cornea, recourse might then be advantageously had to the ingenious proposal of the late Mr. Gibson of Manchester. When the iris was prolapsed, either through a wound or ulcer of the cornea, he found that the displacement arose from the quantity of the aqueous humor lodged behind the iris, constantly pressing that membrane forwards at the point where there was least resistance. In such cases he made a puncture in the prolapsed portion of iris ; and when the fluid collected behind it was discharged, the iris immediately regained its natural situation; unless in those cases, where, from the duration of the disease, the situation of the iris had become unalterable, adhesions having formed between it and that portion of the cornea with which it was in contact. 
\$11. Of the Effects of evacuating the Aqueous Humor, in Injuries of the Eyje-ball.

Injuries of the eye-ball are very often followed by severe and tedious attacks of inflammation, more particularly punctured wounds, and wounds penetrating into any of the cavities.

Besides a powerful antiphlogistic treatment, much benefit will often be derived by discharging the aqueous humor, especially in those cases where, along with pain and redness of the eye, there is a sense of fullness and dull pain about the eyebrow, or some other part of the head;-symptoms of ophthalmia, which, it has already been remarked, the evacuation of the aqueous humor is particularly well calculated to remove. In all cases, too, where there is a tendency to the formation of matter after an injury of the eye, the discharge of the aqueous humor becomes a powerful auxiliary to the other means usually employed.

\section{CASE X.}

At the union of the cornea and sclerotica of the eye of a middle-aged woman, towards the upper and nasal part, there was a prominent bluishcoloured tumor covered with a network of red vessels, and the iris was drawn towards that part, 
so that the pupil was of an oblong form. There were a good number of red vessels over the sclerotic coat, and a dimness of the whole anterior chamber. She complained of much pain in the eyeball, but particularly in the brow and temples. Her sight was impaired, and the pulse frequent and full. About eight days before, she had received a wound of the eye either from the spur or beak of a cock.

All the symptoms were alleviated by the discharge of the aqueous humor, particularly the pain in the head, which was instantly removed; and the redness of the conjunctiva was much diminished. A slight heaviness about the eyebrow remained for a day or two, but went off by the use of brisk purgatives.

\section{CASE XI.}

While a strong healthy man was employed hammering melted iron, a piece of it fell into the eye, and was found lying between the eye-ball and under eye-lid. It occasioned most excruciating pain, and although it was removed in a few minutes, violent inflammation succeeded, and I saw him four days after the accident. The whole conjunctiva was then very much inflamed, and it was so much swelled, that the cornea appeared as if de. 
pressed. There was a white slough towards the inferior part of the sclerotic coat, and on the corresponding portion of the internal palpebral membrane; but the cornea was not injured, nor its transparency diminished. He complained of great pain in the ball of the eye, extending over the forehead, and through the whole side of the head. He could not raise the upper eye-lid without the assistance of his finger. Light gave considerable uneasiness, and his vision was so much destroyed, that he could only distinguish between light and darkness.

Treatment.-Under these circumstances I discharged the aqueous humor by "making a small opening through the transparent cornea. The operation occasioned a smarting pain, which lasted a few seconds; when it went off, he said he could open the eye-lid much easier; and was surprised to find that he could even distinguish the furniture of the room and books in a library. Slight scarifications were afterwards made on the under eye-lid, which bled freely; and he was advised to foment the temples and adjacent parts; and as his pulse was frequent and full, he was bled at the arm, and ordered a brisk purge. During the remaining part of the day, the pain of the eye-ball was much alleviated, and that of the head was completely removed, except that an uneasy sensation still remained in the brow. On the following day there was no vestige of the wound of the cornea; the 
pain and swelling of the conjunctiva were nearly gone, but the redness continued. His vision was quite distinct, but the eye was irritable. In three days, by the application of an opiate, the inflammation was completely removed, his vision perfectly restored, and he returned home complaining merely of a little tenderness. Since which he has remained well.

A case of inflammation of the eye, occasioned by a burn, exactly similar to the case just related, though the symptoms were not so severe, was completely relieved by the evacuation of the aqueous humor, which suddenly mitigated the pain, and removed all the inflammatory symptoms.

5 12. Of the Changes produced in Opacities of the Cornea from the Discharge of the Aqueous. Humor.

It has already been observed that a diminution may take place in the transparency of the cornea, from an alteration in the quantity of the contents of the eye-ball; and it has also been noticed, that in the dead eye the cornea assumes a milky colour when pressure is applied upon the eye-ball, or when the veins are injected with pure water.

That some Opacities of the cornea are produced 
from an increase in the quantity of the contents of the eye-ball, and not from the disposition of an albuminous fluid in the texture of the cornea, as takes place in common speck, seems to be proved from the immediate effects which have resulted, in some instances, from the discharge of the aqueous humor. In these cases the opacity of the cornea seemed entirely to arise from over-distension; for the instant the aqueous humor was discharged, the cornea regained its natural transparency, as imitated in the experiment on the dead eye. In other cases it was evident that the opacity of the cornea depended on two distinct causes; the one, as in the former cases, arising from over-distension, - whilst the other proceeded from that change in the structure of the cornea which produces common speck. Cases of the first class are distinguished by a cloudiness or turbid state of the whole anterior chamber; whereas in common speck the obscurity is more defined, being limited to a certain portion of cornea.

These two different kinds of opacity were strikingly illustrated, in some instances where both took place in an eye at the same time, there being a general dimness throughout the whole anterior chamber, besides some defined $d^{\prime}$ spots in particular parts of the cornea. In these cases, the instant the aqueous humor was discharged, all the general obscurity disappeared, and nothing remained but the more opake spots, which became more distinctly circumscribed. 
From what has already been said, little diffieulty will arise in selecting those cases of opacity of the cornea, which the evacuation of the aqueous humor is calculated to remedy. From the cases, the particulars of which are detailed, the beneficial effects of the operation are sufficiently obvious, and would lead us to expect very important results from future experience.

\section{CASE XII.}

In a girl, twelve years of age, the whole of the white of the right eye-ball had become of a bright red colour, from a number of blood-vessels, whose trunks could all be separately distinguished running in straight lines towards the cornea, and some of their small branches passed over its edge, and were distributed into its substance. There was a circular-shaped speck, of a considerable size, near the centre of the cornea; and throughout the rest of the cornea, particularly around the speck, there was a good deal of muddiness. The upper eye-lid was slightly swelled, with some varicose veins on its external surface ; whilst the vessels of the internal membrane were increased in number and in size. She had a good deal of pain in the eye-ball, but it was particularly severe in the side of the head and temple above the affected eye. Though the vision was destroyed, yet she complained when 
the eye was exposed to a bright light. The disease had continued three weeks. It began with the sensation of a mote in the eye, attended with pain; the following morning it was very red, and every symptom had daily increased.

Treatment.-The aqueous humor was discharged, which caused a good deal of smarting, but in a few minutes that subsided. A great change took place in the transparency of the cornea, all the general cloudiness instantly going away, and the speck left much more distinctly circumscribed. During the course of the day the pain of the head and eye went off, and she slept well during the night, whereas formerly her nights were very restless and disturbed by startings. On the day subsequent to the operation, she had not the least pain in the head or eye; the eye was not very sensible to light, and the number of blood-vessels was very much diminished. She could now readily distinguish all her fingers, and observe large objects at a considerable distance.

\section{CASE XIII.}

The eye-ball of a woman, thirty-eight years of age, was very much inflamed, and some spots of a puriform fluid were seen in the anterior chamber, whilst, at the same time, there was a con- 
siderable degree of opacity throughout the cornea. She complained of an intense pain of the eye-ball, and in the forehead and temples. The disease was of three weeks' standing.

Treatment.-The aqueous humor was discharged, the parts were afterwards fomented, and she took a brisk purge. On the following day the pain of the head was much easier, and the blood-vessels were less numerous.--She rapidly got well.

\section{CASE XIV.}

A lady, thirty-two years of age, had complained during eleven weeks of an inflammation in her right eye, attended with pain occasionally in the forehead, and inability to look at objects. There was a very opake speck of the cornea opposite the pupil, with a degree of turbidness around it, so that nearly the whole pupil was hid; the white of the eye was slightly red, and the eye-lids were of a purple colour towards the edges; the eye was watery. Scarifications, opiates, and stimulants, gave no relief.

Treatment.-The aqueous humor was discharged, and instantly the whole speck, except a small central portion, disappeared, and she could distinctly see with this eye immediately afterwards. All the inflammatory symptoms subsided in a few days by fomentations. 


\section{$C A S E X V$.}

The whole of the white of the eye of a healthy middle-aged man was crowded with vessels of a large size, and of a bright red colour : these vessels all terminated at the margin of the cornea, ran in a straight direction towards it, and did not ramify until they approached it, and there they divided into numerous branches. The transparency of the anterior chamber was diminished, and all objects appeared dim. The eye-lids were a little swelled, and the blood-vessels on their internal surface, though not much increased in number, were enlarged and turgid. He had a violent pain in the forehead, which was not constant, but came on in such severe paroxysms that he could scarcely support their violence. The inflammation had come on four days before without any evident cause.

Treatment.-When the aqueous humor was discharged, the anterior chamber acquired its natural transparency, and he could more clearly distinguish abjects. A remarkable change took place in the blood-vessels of the white of the eye; the number of them was so much diminished, and also their size, that the eye at once lost all the appearances of inflammation.

Besides ordering a purge, and applying fomenta- 
tions to the eye, it was thought proper to bleed this patient, from the violence of the inflammation and its accompanying fever. Under that treatment he got well rapidly.

Two Cases of Ulcers in the Cornea, where the Aqueous Humor was advantageously discharged.

\section{CASE XVI.}

In a young lady the anterior chamber of one eye appeared very turbid, and there was an Ulcer on the central part of the cornea, and a cluster of blood-vessels passing towards it; the whole eyeball was much inflamed, having the peculiar redness of the pustulous ophthalmia*. She complained of an agonizing pain in the forehead, which sometimes went off during the day, but was always severe in the night. The inflammation had lasted fifteen days, the pain in the head only eight days ; little sleep, pulse quick, and the tongue white.

Treatment.-Discharged the aqueous humor by puncturing the cornea at the place where the vessels passed. The pain in the head never afterwards returned; and all the other symptoms rapidly subsided by the use of fomentations, and the vinous tincture of opium.

* See Essay on the Morbid Anatomy of the Eye. 
190 Of EVACUATING THE AQUEOUS humor, \&C.

\section{CASE XVII.}

A healthy looking young man had an Ulcer of the cornea, accompanied with a good deal of inflammation and pain in the eye-ball, in consequence of the suppuration of a pustule. The ulceration and inflammation subsided rapidly by the use of the vinous tincture of opium.

A few days after the eye had recovered this attack, he was suddenly seized with acute pain in it, which soon extended to the head. When I saw him, three days after its commencement, there was a distinct erosion, in two or three different places, with a good deal of muddiness of the cornea. There was also a bright redness on the white of the eye; he had intense pain in the head, accompanied with excessive languor and debility; his tongue white, and a quick hard pulse. The obscurity of the cornea instantly disappeared by the evacuation of the aqueous humor, and the pain of the head was alleviated. He was bled at the arm profusely, and the eye was fomented. On the following day all the symptoms were much relieved; and in a few days the ulcer healed, and the eye recovered perfectly, without the aid of any local applications. 